\title{
COMPORTAMENTO TÉRMICO-REOLÓGICO DE XAROPES COMPOSTOS POR MEL E EXTRATOS NATURAIS.
}

\author{
Lorrayne O. Nascimento' \\ Juliano R. Pereira"l \\ Clarisse L. de Assis'II \\ Marco T. R. Alves ${ }^{\mathrm{IV}}$ \\ Pâmela S. Silvav \\ Rodrigo L. Fabrivı \\ Leonardo M. Mendonça ${ }^{\mathrm{VII}}$ \\ Jéferson G. Da Silva VIII \\ Ângelo M. L. Denadai'x.
}

\begin{abstract}
O presente trabalho teve como objetivo avaliar o comportamento térmico/reológico de três diferentes composições fitoterápicas à base de mel e extratos naturais (denominadas amostras I, II e III), comercializadas na região de Governador Valadares - MG. Os espectros de infravermelho dos xaropes apresentaram grandes similaridades entre se a amostras puras de mel, sugerindo qualitativamente composição química semelhante. Do ponto de vista físico-químico, as amostras I e II apresentaram comportamentos bem similares, com valores aproximados de massa seca, $\mathrm{pH}$, brix, índice de refração e tendência de ionização. As amostras I e II apresentaram comportamento quase newtoniano para um ciclo ascendente-descendente de cisalhamento. A amostra III, de maior massa seca, foi a que apresentou maiores valores de viscosidade aparente além de pseudoplásticidade e histerese reológica, os quais foram atribuídos à presença de estrutura tridimensional do líquido. Quando diluídas em água, apesar da brusca queda da viscosidade, as três amostras assumiram comportamento dilatante, devido à espontânea formação de nanoestruturas iônicas deformáveis, cujo tamanho se reduz com o aumento de temperatura.
\end{abstract}

PALAVRAS CHAVE: XAROPES COMPOSTOS, MEL, EXTRATOS NATURAIS, COMPORTAMENTO TÉRMICO, COMPORTAMENTO REOLÓGICO.

Universidade Federal de Juiz de Fora, UFJF, Campus Governador Valadares. Departamento de Farmácia. Av. Dr. Raimundo Monteiro de Rezende, 330, Centro, Governador Valadares - MG. CEP: 35010-177, Brasil.

'Discente do Curso de Graduação em Farmácia, Universidade Federal de Juiz de Fora, Campus Governador Valadares, MG, Brasil (e-mail: lorrayne.on@hotmail.com).

"Engenheiro de Alimentos, Técnico Administrativo em Educação do Departamento de Farmácia, Universidade Federal de Juiz de Fora, Campus Governador Valadares, MG, Brasil (e-mail: juliano.rocha@ufj.edu.br).

'"'Discente do Curso de Graduação em Farmácia, Universidade Federal de Juiz de Fora (UFJF), Campus Governador Valadares, MG, Brasil (e-mail: clarisseieq@hotmail.com).

IVDiscente do Curso de Graduação em Farmácia, Universidade Federal de Juiz de Fora (UFJF), Campus Governador Valadares, MG, Brasil (e-mail: marco_tullioalves@hotmail.com).

vDoutora em Ciências Médicas, Departamento de Farmácia, Universidade Federal de Juiz de Fora (UFJF), Campus Governador Valadares, MG, Brasil (email: pamela.souza@ufjf.edu.br).

V'Doutor em Ciências Biológicas, Departamento de Farmácia, Universidade Federal de Juiz de Fora (UFJF), Campus Governador Valadares, MG, Brasil (email: rodrigo.fabri@ufjf.edu.br).

V"Doutor em Toxicologia, Departamento de Farmácia, Universidade Federal de Juiz de Fora (UFJF), Campus Governador Valadares, MG, Brasil (email: leonardo.mendonca@ufjf.edu.br).

VII'Doutor em Química, Departamento de Farmácia, Universidade Federal de Juiz de Fora (UFJF), Campus Governador Valadares, MG, Brasil (email: jefersongomes.silva@ufj.edu.br).

'xDoutor em Química, Departamento de Farmácia, Universidade Federal de Juiz de Fora (UFJF), Campus Governador Valadares, MG, Brasil (email: angelo.denadai@ufjf.edu.br). 


\section{INTRODUÇÃO}

O mel é uma mistura fluida natural, de elevada complexidade, produzida pelas abelhas a partir de néctar de plantas ou de misturas de açúcares segregados por insetos. Do ponto de vista químico, é rico em açúcares (70-80\%), contendo de 10 a $20 \%$ de água. Ele pode também apresentar vitaminas, minerais, compostos fenólicos, aminoácidos livres e enzimas; de modo que as concentrações relativas desses constituintes são extremamente dependentes da espécie de abelha produtora, origem floral geográfica e condições climáticas (Nagai et al., 2006; Bogdanov et al., 2008). Além de sua qualidade como alimento, esse produto único é dotado de inúmeras propriedades terapêuticas, sendo utilizado pela medicina popular sob diversas formas e associações fitoterápicas, com esperada ação antimicrobiana, antiviral, antioxidante, antitumoral e anti-inflamatória (ViudaMartos et al., 2008).

A viscosidade é uma das propriedades mais importantes do mel, sendo um parâmetro físicoquímico que interfere diretamente nas suas características sensoriais. As propriedades reológicas do mel são influenciadas por vários fatores tais como a temperatura, composição química (teor de açúcares, compostos essenciais, porcentagem de $\mathrm{H}_{2} \mathrm{O}$ ) e a quantidade e tamanho de cristais (White, 1978). Assim, o conhecimento e controle do comportamento reológico são indispensáveis para dimensionamento de equipamentos nas diversas operações de processamento de materiais, misturas e composições alimentícias contendo mel.

Como a composição química interfere diretamente nas interações intermoleculares entre os componentes do mel e seus derivados, diferentes composições inevitavelmente apresentarão diferentes perfis reológicos. Além disso, misturas fitoterápicas de amostras de mel com extratos vegetais, na forma de xaropes, por tornarem a composição ainda mais complexa, podem gerar efeitos imprevisíveis na viscosidade da composição.

Considerando o fato de que xaropes fitoterápicos comerciais são misturas cuja composição química varia de fabricante para fabricante e que a viscosidade é um importante parâmetro sensorial que pode mudar sob diversas condições de temperatura e cisalhamento durante a produção, armazenamento e transporte; o presente trabalho teve como objetivo a investigação das propriedades reológicas de amostras de xarope obtidos de três diferentes fornecedores da região de Governador Valadares - MG. Como a composição dos xaropes não foi um dado acessível no presente estudo, as características reológicas foram correlacionados com os parâmetros físicoquímicos $\mathrm{pH}$, condutividade, brix, índice de refração e \% de massa seca. Espectros de infravermelho foram também obtidos para investigação qualitativa da composição química. Finalmente, a presença de estruturas coloidais nos xaropes e sua dependência da temperatura foram investigadas por experimentos de espalhamento de luz dinâmico (DLS).

\section{MATERIAIS E MÉTODOS}

\subsection{AMOSTRAS}

No presente estudo foram utilizadas amostras constituídas por mel e extratos naturais adquiridas comercialmente de três fornecedores da cidade de Governador Valadares - MG, sendo elas denominadas $I_{p}, I_{p}$ e $I I I_{p}$, onde o índice "p" indica se tratar de amostras puras. As denominações $I_{d}, I_{d}$ e III $I_{d}$ foram utilizadas para se referir às amostras diluídas na proporção volumétrica de 1:1. Tais amostras foram utilizadas sem nenhum tratamento prévio além de diluição em água destilada, quando necessário. Além da denominação "Xarope de Mel e Guaco", nenhuma outra informação relativa à composição dos xaropes, tais como concentrações relativas de mel e extratos, teor de água ou de qualquer outro constituinte constavam no rótulo das amostras. Portanto, procedeu-se com as caracterizações físico-químicas sem o conhecimento exato da composição das amostras. 


\subsection{CARACTERIZAÇÕES FÍSICO-QUÍMICAS}

Os espectros na região do infravermelho médio foram obtidos utilizando um acessório de refletância total atenuada (ATR) da marca PIKE acoplado a um espectrômetro Perkin Elmer Spectrum Two ${ }^{T M}$. Após a aplicação direta de cada amostra pura $\left(I_{p}, I_{p}\right.$ e $\left.I I_{p}\right)$ sobre superfície de diamante do modulo de ATR, os espectros foram obtidos como a média de 16 varreduras consecutivas, com resolução de $2 \mathrm{~cm}^{-1}$ e adquiridos em um intervalo de número de onda 4000 a $600 \mathrm{~cm}^{-1}$. Para a aquisição dos espectros foi utilizado o programa Perkin Elmer Spectrum ES (versão do aplicativo: 10.03.08.0133).

A massa seca das amostras (sólidos não voláteis) foi determinada por análises gravimétricas, com secagem $a \approx 100{ }^{\circ} \mathrm{C}$. $\mathrm{O}$ índice de refração e Brix foram determinados em um refratômetro Instrutherm (modelo RTD-95). Medidas de $\mathrm{pH}$ foram realizadas em um potenciômetro HANNA (modelo modelo HI3221). Medidas de condutividade elétrica foram realizadas em um condutivímetro TECNAL (modelo Tec 4MP). Ambas as medidas foram realizadas por imersão direta dos eletrodos nas amostras sem nenhum tratamento, após prévia calibração dos equipamentos. Medidas de condutividade elétrica foram também utilizadas para avaliar o grau de ionização dos componentes das amostras. Para isso, foram preparadas 10 diferentes frações volumétricas de xarope, variando de $X_{\text {xar/H2O }}=0$ (100\% de água) a $X_{\text {xar/H2O }}=$ 1 (100\% de xarope), as quais foram submetidas a medidas de condutividade. Os valores foram plotados em um gráfico de $k \times X_{\text {xar/H2O }}$. O potencial de ionização $(P I-\mu S / c m)$, proporcional ao número de sítios ionizáveis dos constituintes das amostras, foi determinado por integração das curvas, conforme equação 1 .

\subsection{ANÁLISES REOLÓGICAS}

O comportamento reológico das amostras puras e diluídas foi avaliado em função da temperatura e da taxa de cisalhamento $(\phi)$, com um reômetro rotacional estacionário de taxa de cisalhamento controlada, marca ProRheo, modelo R-180. Para construção das curvas de temperatura, as amostras foram aquecidas de $\approx 30^{\circ} \mathrm{C}$ até $\approx 80^{\circ} \mathrm{C}$, a uma taxa de cisalhamento $(\phi)$ constante de $500 \mathrm{~s}^{-1}$, dentro do limite de detecção reológica do equipamento. Para a construção das curvas de fluxo e de viscosidade, as amostras foram pré-cisalhadas a $\phi=100 \mathrm{~s}^{-1}$ por $24 \mathrm{~s}$ e submetidas a uma variação cíclica de taxa de cisalhamento, no intervalo de $100<\phi<1000 \mathrm{~s}^{-1}$, nos sentidos ascendente e descendente.

\subsection{ESPALHAMENTO DE LUZ DINÂMICO (DLS)}

O diâmetro hidrodinâmico das nanoestruturas coloidais foi medido por espalhamento de luz dinâmico em um fotômetro MalvernZetasizer Nano ZS90. As medidas foram feitas em função da temperatura com controle Peltier, no intervalo de $20^{\circ} \mathrm{C}$ a $80^{\circ} \mathrm{C}$, com incrementos de $5^{\circ} \mathrm{C}$ cada $(12$ pontos no total).

As amostras foram diluídas em água na proporção volumétrica de 1:1 e inseridas em cubeta de vidro de $1 \mathrm{~cm}$ de caminho óptico, padrão Malvern, específica para varredura de temperatura. As suspensões foram então submetidas a um feixe de luz monocromático (Laser He-Ne de $4 \mathrm{~mW}$ e comprimento de onda de $633 \mathrm{~nm}$ ) e a intensidade de luz espalhada foi medida a um angulo de $90^{\circ}$. O Diâmetro hidrodinâmico $\left(D_{h}\right)$ foi determinado pela média de três medidas independentes, cada uma delas obtida a partir da média de 5 contagens. 


\section{RESULTADOS E DISCUSSÃO}

\subsection{CARACTERIZAÇÃO FÍSICO-QUÍMICA DAS AMOSTRAS}

Apesar do desconhecimento das concentrações e composições dos diferentes aditivos adicionados em cada uma das amostras, os três xaropes apresentaram espectros de infravemelho muito semelhantes entre si (Figura 1), típicos de amostras puras de mel (Bertelli et al., 2007; Svečnjak et al., 2011). Nesses espetros foram observadas bandas em torno de 3290, 2932 e 1644 $\mathrm{cm}^{-1}$ atribuídas aos estiramentos da ligação $\mathrm{O}-\mathrm{H}$ das moléculas dos carboidratos (frutose, maltose e sacarose) e da água, estiramentos da ligação $\mathrm{C}$-H presentes nas moléculas dos carboidratos e deformação angular da molécula de água, respectivamente (Svečnjak et al., 2011). Além disso, as bandas encontradas na região de 1500 a $600 \mathrm{~cm}^{-1}$ estão associadas às ligações que formam o esqueleto dos carboidratos. Nenhuma banda diferente daqueles comumente encontradas em amostras puras de mel foram encontradas (Bertelli et al., 2007; Svečnjak et al., 2011). Essa informação é coerente com o baixa quantidade de extrato de guaco nas amostras.

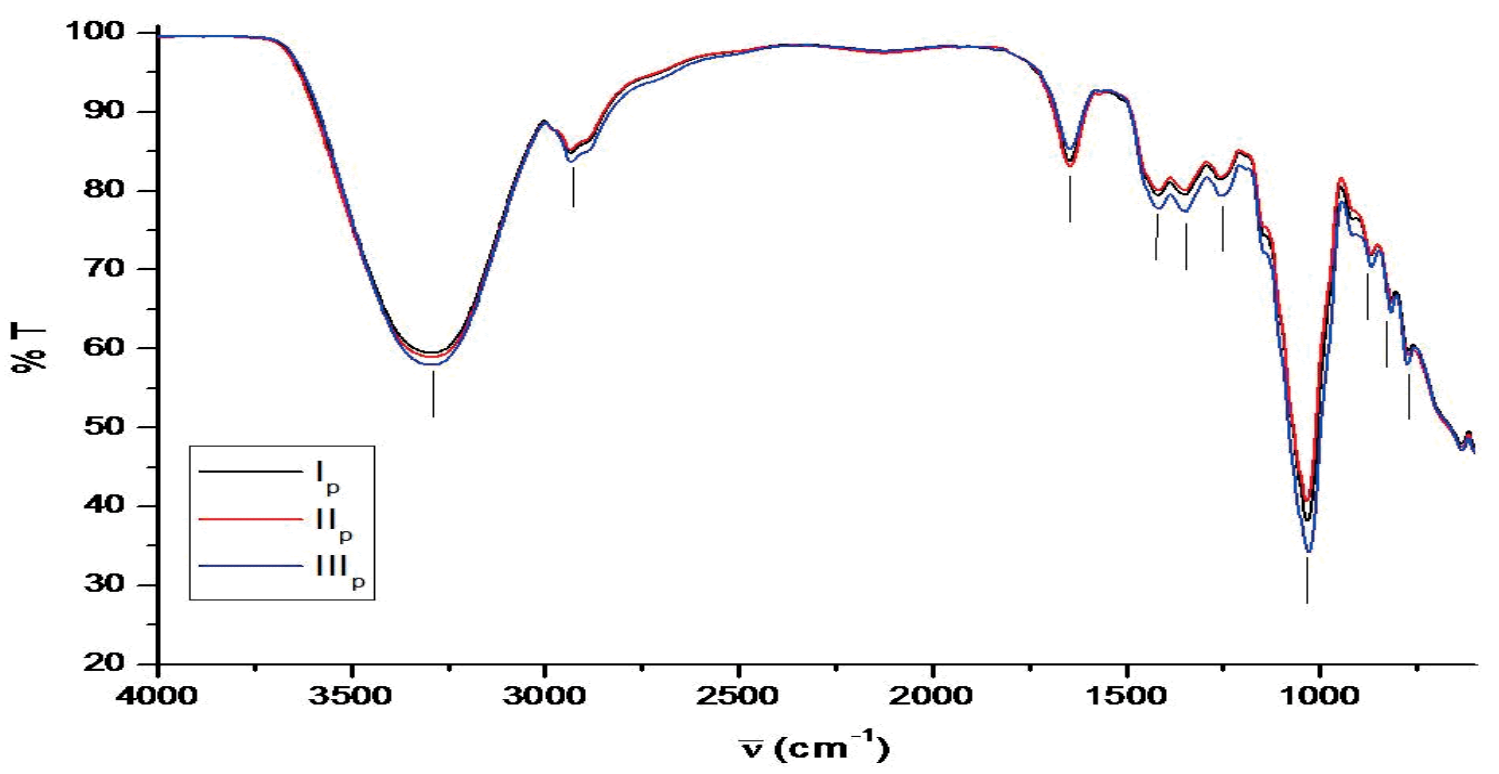

FIGURA 1: ESPECTROS NA REGIÃO DO INFRAVERMELHO MÉDIO PARA AS TRÊS
AMOSTRAS DE XAROPES À BASE DE MEL UTILIZADOS NESTE TRABALHO.

As amostras puras foram então submetidas a uma série de análises físico-químicas complementares cujos resultados são apresentados na Tabela 1. Conforme resultados, as composições são ácidas $(\mathrm{pH} \approx 4)$ e pouco condutoras. Esses dados são condizentes com a presença de ácidos fracos não ionizados (Silva et al., 2009; de Almeida-Muradian et al., 2014).

TABELA 1: PARÂMETROS FÍSICO-QUÍMICOS DOS TRÊS XAROPES

\begin{tabular}{ccccccc}
\hline & $\% \mathbf{m}_{\text {seca }}$ & $\mathbf{p H}$ & $\mathbf{P I}(\mu \mathrm{S} / \mathbf{c m})$ & $\mathbf{k}_{\mathrm{p}}(\mu \mathrm{S} / \mathbf{c m})^{*}$ & Brix & $\mathbf{I R}^{\star *}$ \\
\hline Amostra I & $75,3 \pm 2,5$ & 3,9 & 259 & 28,04 & $72,7 \pm 0,9$ & $1,471 \pm 0,001$ \\
\hline Amostra II & $72,6 \pm 4,8$ & 4,0 & 189 & 12,37 & $79,97 \pm 0,06$ & $1,490 \pm 0,000$ \\
\hline Amostra III & $86,0 \pm 3,0$ & 3,9 & 502 & 10,97 & $73,3 \pm 0,9$ & $1,473 \pm 0,003$ \\
\hline
\end{tabular}

${ }^{*} k_{p}(\mu S / c m)$ : valores de condutividade para as amostras puras; ${ }^{*} \mid \mathrm{R}$ : índice de refração; Condutividade da água utilizada nos experimentos: $\mathrm{k}_{\mathrm{H} 2 \mathrm{O}}=6 \mu \mathrm{S} / \mathrm{cm}$. 
Os valores de brix encontrados para os xaropes foram próximos daqueles encontrados para amostras puras de mel de diferentes países (da ordem de $80^{\circ}$ ) (Bhandari et al., 1999; Silva et al., 2009). Das três amostras, a III $I_{\mathrm{p}}$ foi a que apresentou maior massa seca. Entretanto, não foi encontrado uma correlação direta entre esse parâmetro com o parâmetro óptico brix e com os espectros na região do infravermelho médio.

O perfil de ionização dos constituintes do xarope foi avaliado pela análise das curvas de $k x X_{\text {xar/H2O }}$, com fração volumétrica de xarope variando de $0 \leq X_{\text {xar/H2O }} \leq 1$ (Figura 2). As três curvas mostraram um significativo aumento da condutividade com a diluição das amostras até $X_{\text {xar/ } / 2 O}=$ 0,3 (30\% de xarope $+70 \%$ de água), o que é condizente com a ionização ácida. O xarope III $_{\mathrm{p}}$ foi o que apresentou maior potencial de ionização $(P I-\mu S / \mathrm{cm})$, atribuído à maior massa seca e, possivelmente, à maior concentração de espécies ácidas.

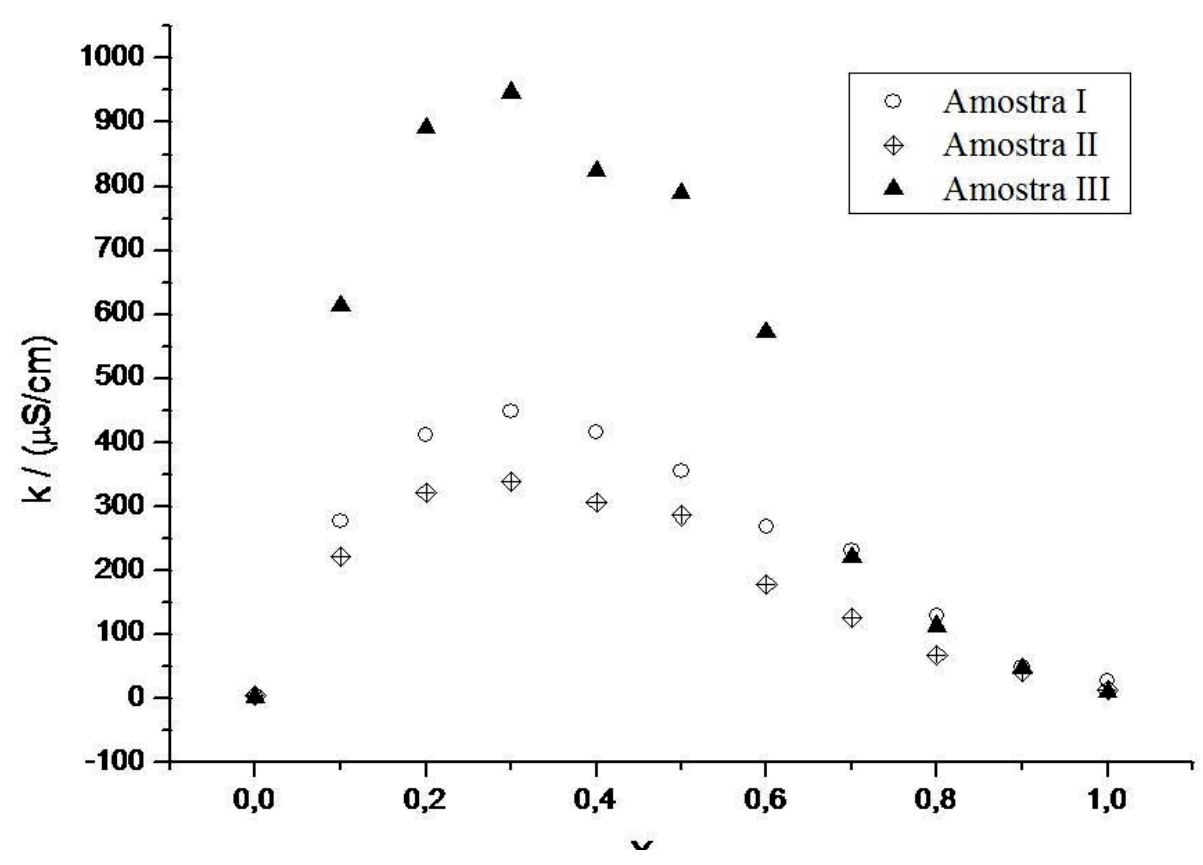

\section{FIGURA 2: CURVAS DE CONDUTIVIDADE EM FUNÇÃO DA FRAÇÃO VOLUMÉTRICA DOS XAROPES.}

\subsection{CARACTERIZAÇÃO REOLÓGICA EM FUNÇÃO DA TEMPERATURA}

As análises reológicas foram realizadas em função da temperatura e em função da taxa de cisalhamento, com a finalidade de se mapear a viscosidade das amostras em diversas condições. Além disso, quanto menor a viscosidade de um fluido, menor é a perda de carga durante o escoamento, diminuindo os custos energéticos com bombeamento.

Nas Figuras 3 e 4 são apresentadas respectivamente as curvas de viscosidade em função da temperatura $(\eta \times T)$ para as amostras puras $\left(I_{p}, I_{p}\right.$ e $\left.I I_{p}\right)$ e diluídas a uma fração volumétrica de 1:1 $\left(I_{d}, I I_{d}, I I I_{d}\right)$. Nesse experimento, foi escolhida a taxa de cisalhamento constante de $\phi=500 \mathrm{~s}^{-1}$ por ser da magnitude dos valores geralmente encontrados em misturadores e em tubulações (Barnes et al., 1998). 


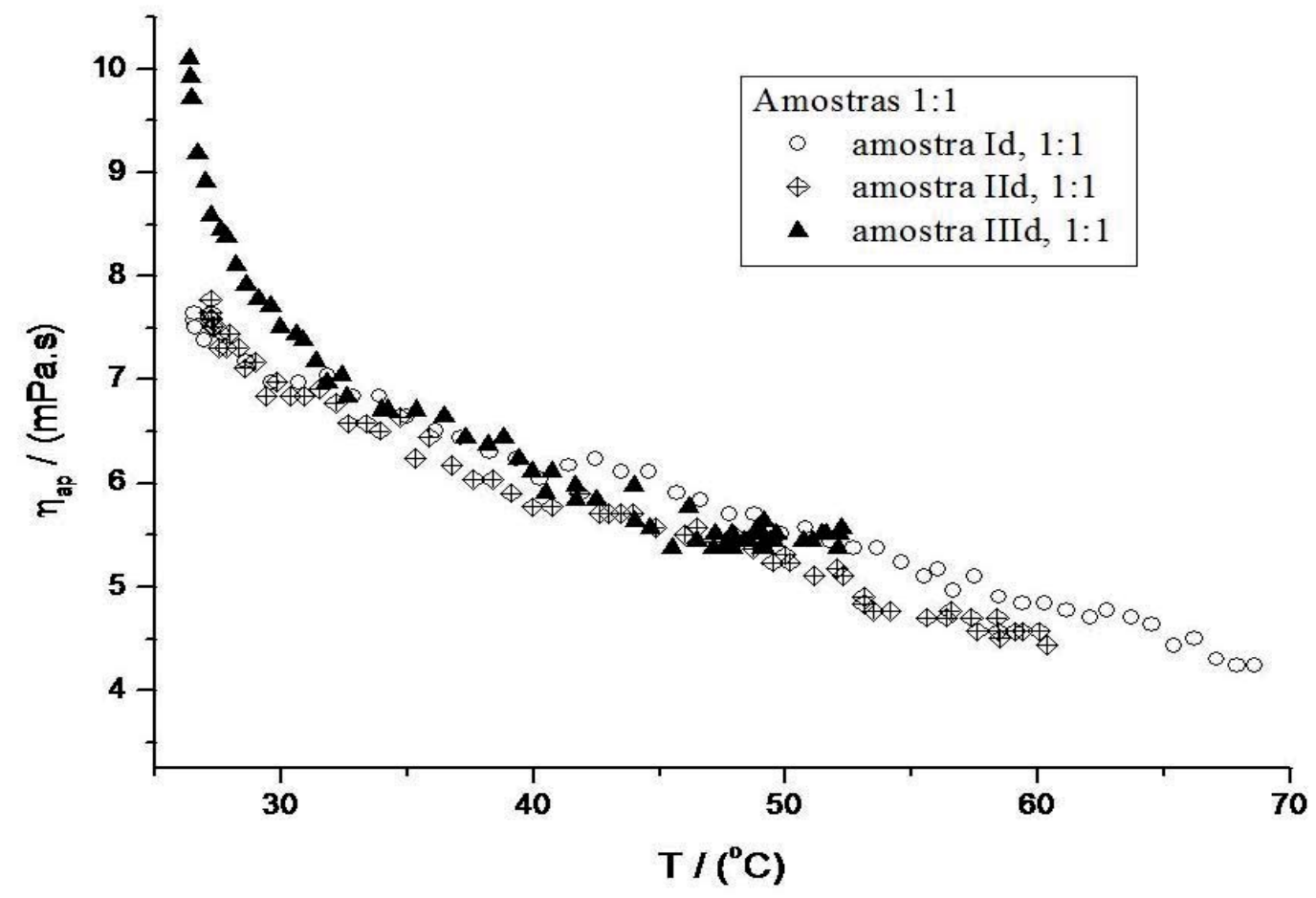

FIGURA 3: CURVAS DE VISCOSIDADE EM FUNÇÃO DA TEMPERATURA PARA AS AMOSTRAS PURAS $\left(I_{P}, I_{P} E I I I_{P}\right)$.

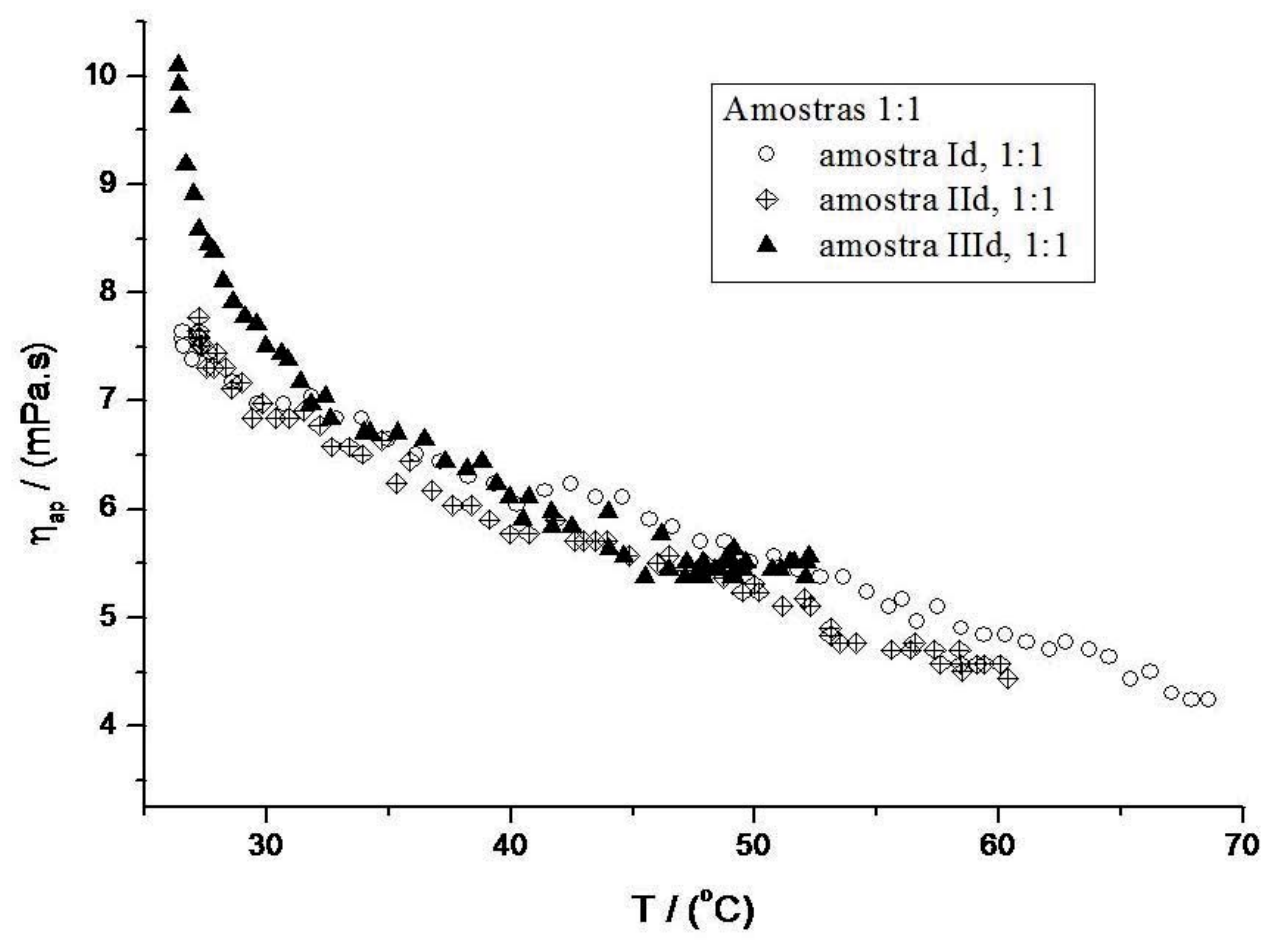

FIGURA 4: CURVAS DE VISCOSIDADE EM FUNÇÃO DA TEMPERATURA PARA AMOSTRAS DILUÍDAS NA PROPORÇÃO 1:1 ( $\left.\mathrm{I}_{\mathrm{D}}, \mathrm{II}_{\mathrm{D}} \mathrm{E} \mathrm{III}_{\mathrm{D}}\right)$. 
As curvas apresentaram comportamento exponencial, característico da quebra de interações intermoleculares com o aumento de temperatura. Isso significa que a uma temperatura de $35^{\circ} \mathrm{C}$ por exemplo, que não é crítica para a estabilidade química das composições, a viscosidade cai de 2 ou 3 vezes, o que facilitaria bastante operações de mistura e transporte. O modelo de Arrhenius (Barnes et al., 1998) (Eq. 2) foi então empregado para ajustar as curvas:

Nessa equação, $\eta_{a p}$ é viscosidade aparente (razão $T / \phi$ ) medida a uma taxa constante de $500 \mathrm{~s}^{-1}, \eta_{\infty}$ é a viscosidade assintótica ou viscosidade à temperatura infinita, $E_{a} / R$ é a energia de ativação de fluxo normalizada pela constante dos gases $(R=8,314 \mathrm{~J} / \mathrm{K})$, dada em K${ }^{-1}$. Cabe ressaltar que quanto mais elevado for o valor de $E_{a} / R$, maior será a sensibilidade da viscosidade com a temperatura devido à existência de uma estrutura tridimensional de interações. Em outras palavras, $E_{a} / R$ é uma medida do grau de estruturação do fluido por interações intermoleculares. A Tabela 2 mostra os valores de $E_{a} / R e \eta_{\infty}$ obtidos através de ajuste não linear da Eq. 2, usando o Microcal Origin Pro 7.0.

Das três amostras puras, o xarope $\mathrm{III}_{\mathrm{p}}$ foi o que apresentou maiores valores de $\eta_{a p}$ e $E_{a} / R$. Sua maior viscosidade foi atribuída à maior massa seca, que por sua vez deve gerar uma maior concentração de interações intermoleculares entre seus constituintes e maior atrito durante o fluxo. O maior valor de $E_{a} / R$ foi interpretado em termos da quebra da estrutura $3 \mathrm{D}$ com o aumento da temperatura.

\section{TABELA 2: DADOS REOLÓGICOS OBTIDOS A PARTIR DA EQUAÇÃO DE ARRHENIUS PARA OS PARÂMETROS: $E_{A} E$ n®; $E$ DE OSTWALD PARA OS PARÂMETROS: N KC E HISTERESE.}

\begin{tabular}{|c|c|c|c|c|c|c|c|}
\hline Amostra & $E_{a} / R\left(K^{-1}\right)$ & $\eta_{\infty}(\mathrm{Pa} . \mathrm{s})$ & $\mathrm{n}_{\mathrm{asc}}$ & $\mathrm{n}_{\text {desc }}$ & $K c_{\text {asc }}\left(P a . s^{n}\right)$ & $K c_{\text {desc }}\left(P a . s^{n}\right)$ & $\mathrm{H}\left(\mathrm{J} / \mathrm{m}^{3} . \mathrm{s}\right)$ \\
\hline Amostra 1 pura & $101,5 \pm 1,7$ & $0,005 \pm 0,000$ & 1,012 & 0,991 & 0,183 & 0,212 & $-856,54$ \\
\hline Amostra 1 1:1 & $24,7 \pm 0,9$ & $0,00314 \pm 0,00008$ & 1,598 & 1,690 & 0,000188 & 0,000104 & 0.01543 \\
\hline Amostra 2 pura & $116,7 \pm 2,0$ & $0,0059 \pm 0,0004$ & 0,997 & 0,986 & 0,370 & 0,393 & 864,01 \\
\hline Amostra 2 1:1 & $23,6 \pm 0,5$ & $0,00321 \pm 0,00004$ & 1,555 & 1,563 & 0,000238 & 0,000226 & 0,02481 \\
\hline Amostra 3 pura & $170,1 \pm 2,8$ & $0,0035 \pm 0,0004$ & 0,898 & 1,016 & 0,394 & 0,171 & 8057,44 \\
\hline Amostra 31-1 & $29,0 \pm 0,8$ & $0,00300 \pm 0,00007$ & 1,487 & 1,535 & 0,000420 & 0,000308 & $-0,02939$ \\
\hline
\end{tabular}

As diferenças nos valores de $n_{\text {asc }}, K c_{\text {asc }} n_{\text {desc }}$ e $K c_{\text {desc }}$ se devem às histereses nas curvas.

Comparando os resultados com os de amostras puras de mel, observa-se que os valores aqui descritos estão da mesma ordem de grandeza. Por exemplo, em amostras puras de mel australiano (Bhandari et al., 1999) foram encontrados valores de $E_{a} / R$ de 149 e $214 \mathrm{~K}^{-1}$. Em amostra nacional (Pereira et al., 2003), tem-se valores de $102 \mathrm{~K}^{-1}$. Isso mostra que a presença dos componentes do extrato, na proporção em que foram inseridos, praticamente não afetam os parâmetros reológicos.

Para as amostras diluídas, observou-se uma brusca queda na viscosidade aparente e uma menor dependência com a temperatura, mostrando que a diluição tende a nivelar o comportamento reológico do mel, independente de sua origem. A pronunciada queda da energia de ativação de fluxo se deve ao rompimento da estrutura tridimensional dos fluidos, provocada pela diluição em água.

\subsection{CARACTERIZAÇÃO REOLÓGICA EM FUNÇÃO DA TAXA DE CISALHAMENTO}

As curvas de fluxo $(\tau \times \phi)$ foram construídas à $30^{\circ} \mathrm{C}$ para amostras puras e diluídas em água (Figuras 5 e 6), sendo então ajustadas segundo o modelo de Ostwald-de-Walle (Barnes et al., 1998) 
Nessa equação, $K_{c}$ é o índice de consistência - a viscosidade do fluido não newtoniano; e $n$ é o índice de comportamento - parâmetro que mede o desvio do comportamento newtoniano (para $\mathrm{n}>1$, tem-se um fluido dilatante; para $\mathrm{n}<1$, tem-se um fluido pseudoplástico e para $\mathrm{n}=1$ tem-se um fluido newtoniano); $\tau$ e $\phi$ são respectivamente a tensão e a taxa de cisalhamento.

A Tabela 2 mostra os valores de $n$ e $K c$ para as amostras puras e diluídas, calculadas para o ciclo de cisalhamento (ascendente e descendente) através da Eq. 3. Também são apresentados valores de histerese de fluxo $\left(H-J / m^{3} s\right)$, calculado por meio da Eq. 4:

Os valores de $n$ mostram que as amostras $I_{p}$ e $I_{p}$ tem comportamento próximo do newtoniano, o que é condizente com o perfil linear das curvas de fluxo. Já a amostra III ${ }_{\mathrm{p}}$ apresentou pseudoplásticidade $\left(n_{\text {asc }}=0,898\right)$ e alta histerese.Tal comportamento é indício de tixotropia, ou seja, dependência temporal da viscosidade em função da existência interações intermoleculares cruzadas (similar a um gel). Durante o cisalhamento, a energia mecânica rompe parcialmente as interações gerando uma transição de gel para sol (Barnes, 1997).

Todas as amostras diluídas apresentaram comportamento dilatante para o ciclo de cisalhamento, com $n \approx 1,5$. Esse fenômeno foi atribuído à presença de micro e/ou nanoestruturas "macias", capazes de sofrer deformação e empacotamento durante o cisalhamento.
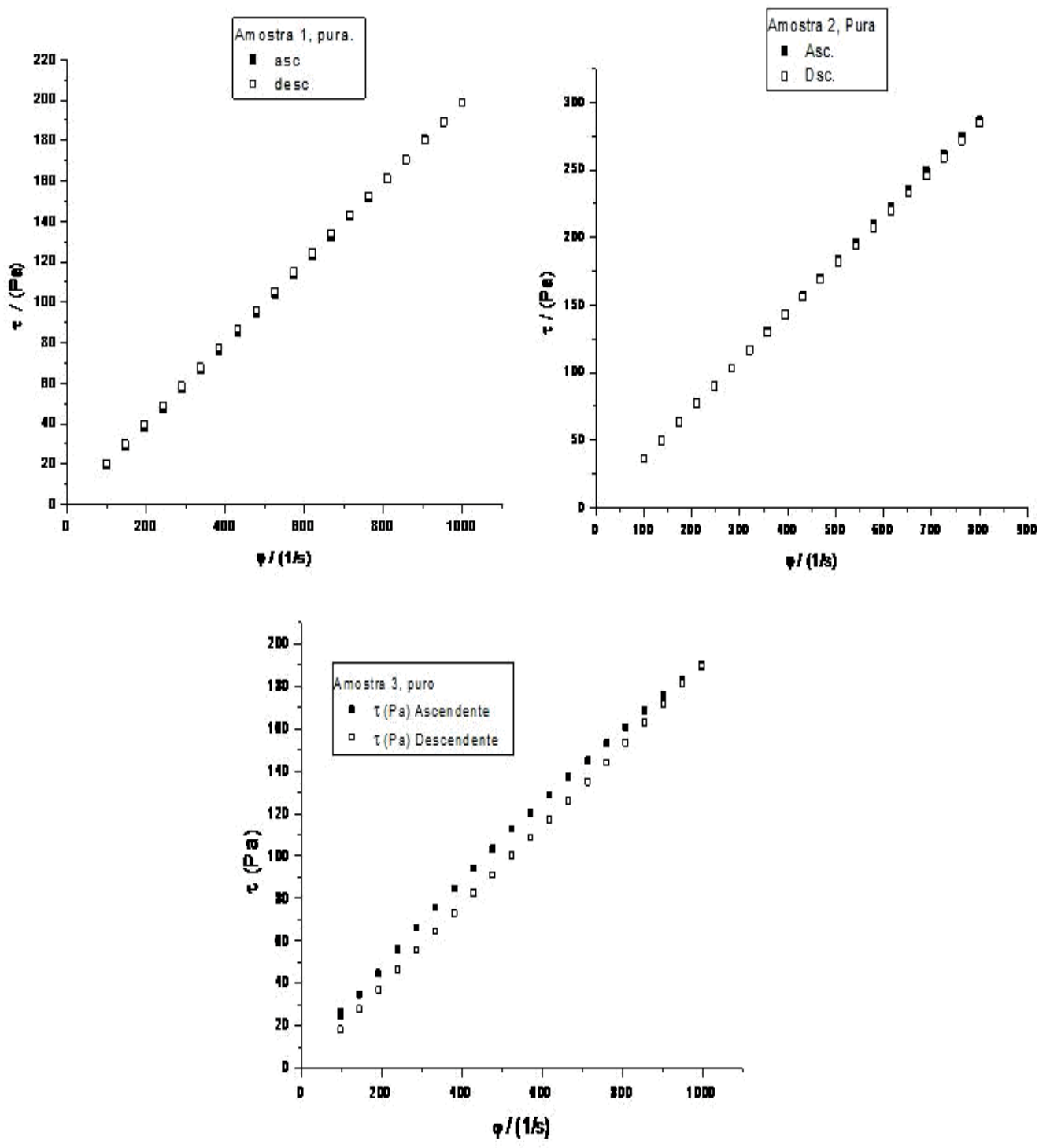

FIGURA 5: Curvas de fluxo das amostras puras. a) Amostra $I_{p}$, b) Amostra II e c) Amostra III 

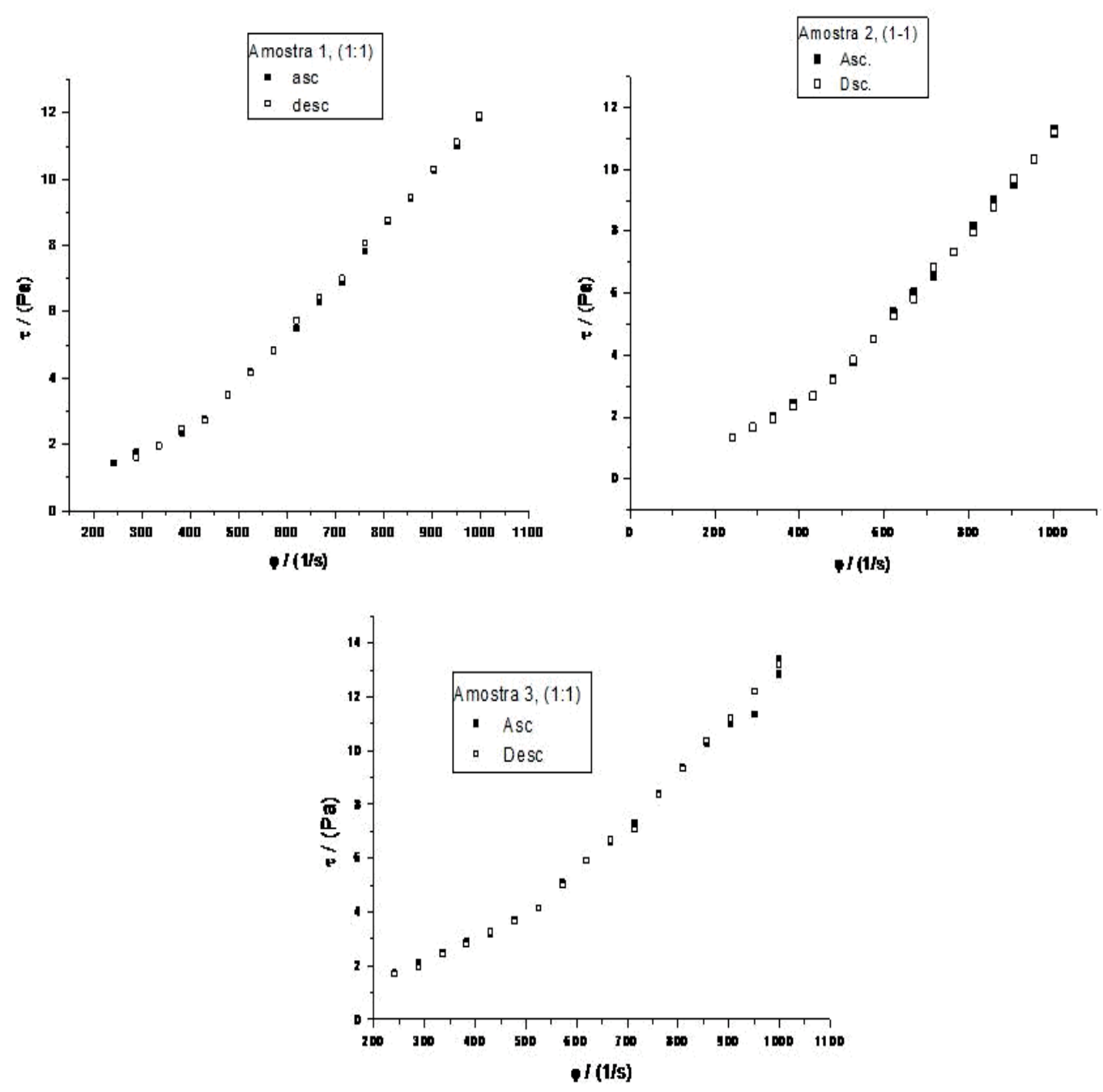

FIGURA 6: CURVAS DE FLUXO DAS AMOSTRAS DILUÍDAS NA PROPORÇÃO DE 1:1. A) AMOSTRA $I_{D}$, B) AMOSTRA II $E$ C) AMOSTRA III.

\subsection{MEDIDAS DE TAMANHO POR DLS}

As três amostras diluídas na proporção volumétrica de 1:1 apresentaram espalhamento Rayleigh, indicando a presença de micro/nanoestruturas dispersas no líquido (Figura 7). É válido salientar que quando as amostras puras foram submetidas ao mesmo experimento, não foram obtidas curvas de correlação (Tseng et al., 2011) que permitissem o cálculo do diâmetro hidrodinâmico. Os tamanhos médios das partículas observadas nas três amostras segue a ordem: $D h_{1}>D h_{\|}>D h_{\| 1}$; embora suas características devam ser similares, já que o perfil reológico dessas amostras com a temperatura foi bastante parecidos (Figura 4).

Como citado, em amostras de mel é comum a presença de nanoestruturas coloidais (Abu-Jdayil et al., 2002). Porém, os dados mostram que no ambiente aquoso a presença dessas estruturas se torna mais perceptível quando ocorre a dispersão em água. Esses estruturas devem ser agregados moleculares de característica anfifílica que, ao sofrerem ionização, atuam como agentes emulsificantes de componentes hidrofóbicos do próprio xarope.

Todas as amostras apresentaram redução do tamanho de partículas com a temperatura. Caso os agregados fossem não-iônicos, um aumento de temperatura levaria a uma desidratação da superfície dos coloides, com uma consequente coalescência e crescimento das estruturas (Balmbra 
et al., 1962; SilvaeLoh, 1998; Hammouda, 2010). Como isso não acontece, tem-se um forte indício de que os agregados sejam iônicos. Comportamento similar é observado em sistemas micelares iônicos, onde o aumento da temperatura favorece a redução do tamanho das micelas por acelerar o movimento Browniano das moléculas, reduzindo o tamanho dos agregados e aumentando a concentração de surfactante livre (Bezzobotnoy et al., 1988; Hammouda, 2013).

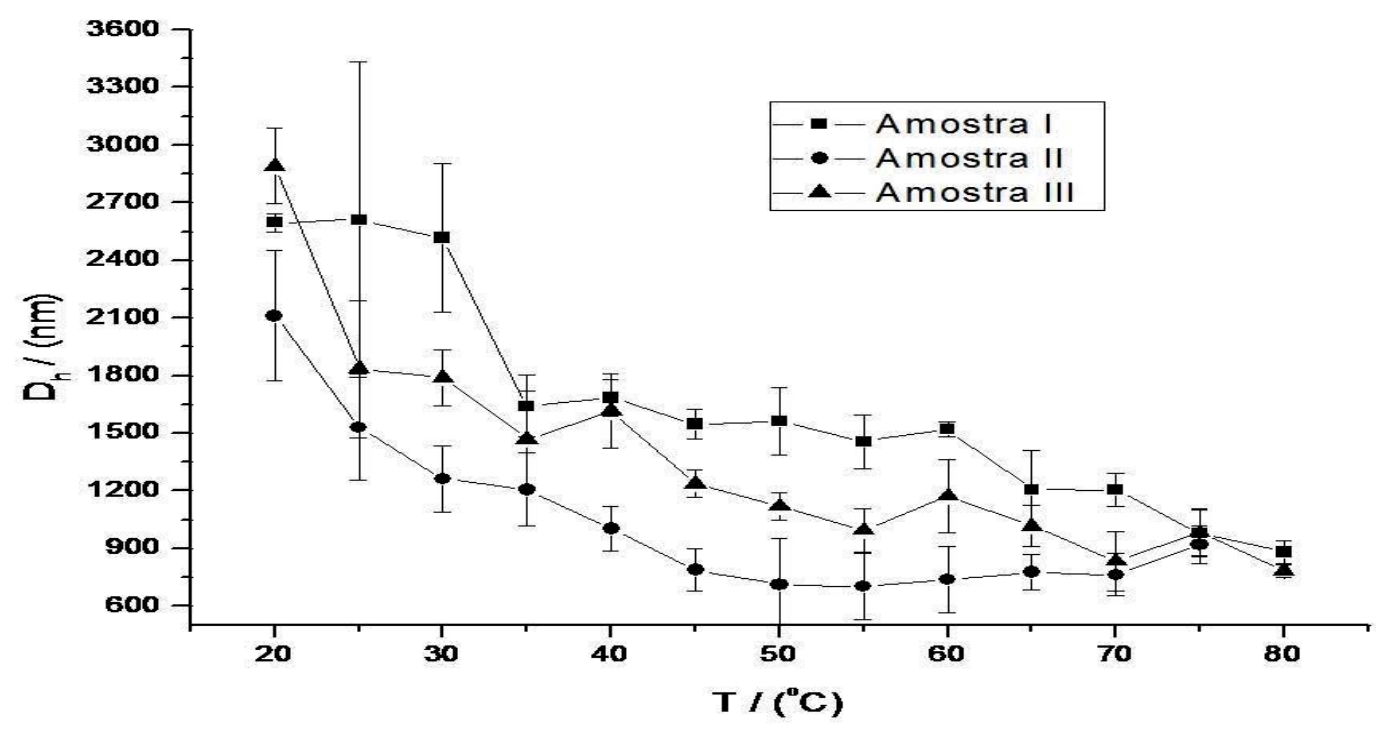

FIGURA 7: DIÂMETRO HIDRODINÂMICO EM FUNÇÃO DA TEMPERATURA.

\section{CONCLUSÕES}

O comportamento reológico de três xaropes à base de mel e extratos de guaco foram avaliadas com respeito à massa seca, $\mathrm{pH}$, condutividade e brix. Para as amostras puras, um ligeiro aumento da temperatura acarretou em uma brusca queda na viscosidade, o que favorece a redução de custos energéticos de mistura e bombeamento. As amostras $I_{p}$ e $I_{p}$ apresentaram comportamento newtoniano enquanto que a amostra III ${ }_{\mathrm{p}}$, de maior massa seca e potencial de ionização, apresentou comportamento pseudoplástico e tixotrópico, atribuídos à existência uma estrutura tridimensional de interações. Após a diluição na proporção volumétrica de 1:1, a viscosidade aparente caiu de 50-100 vezes. Entretanto, as amostras passaram a apresentar comportamento dilatante, devido à formação espontânea de agregados moleculares deformáveis, que por sua vez poderiam atuar como sistemas de liberação controlada dos princípios ativos dos xaropes no meio biológico. A massa seca demonstrou ser um dos mais importantes parâmetros na definição do comportamento reológico das composições.

\section{THERMAL AND RHEOLOGICAL BEHAVIOR OF HERBAL HONEY AND NATURAL EXTRACTS SYRUPS}

\section{ABSTRACT}

This present study aimed to evaluate the rheological thermal behavior three different herbal honey and natural extracts compounds, marketed in the region of Governador Valadares - MG. The 
infrared spectra of the three samples were very similar themselves and to pure samples of honey, suggesting similarity of chemical composition. From a physic-chemical standpoint, the samples I and II showed very similar behavior, with approximate of dry matter, $\mathrm{pH}$, Brix, refractive index and trend of ionization. The samples I and II presented Newtonian behavior for an up-down shear cycle. The sample III, which showed higher dry mass, presented the highest apparent viscosity, rheological hysteresis and pseudoplasticity, which were attributed to the presence of tridimensional structure of the liquid. When diluted in water, in spite of the drastic reduction of viscosity, the three samples taken dilatant behavior due to the spontaneous formation of ionic and soft nanostructures, with decreasing size with increasing of temperature.

KEYWORDS: COMPOUND SYRUPS, HONEY, NATURAL EXTRACTS, THERMAL BEHAVIOR, RHEOLOGICAL BEHAVIOR.

\section{REFERÊNCIAS BIBLIOGRÁFICAS}

1 ABU-JDAYIL, B.; GHZAWI, A.A.M.; AL-MALAH, K.I.M.; ZAITOUN, S. Heat effect on rheology of light- and dark-colored honey. Journal of Food Engineering, v. 51, n. 1, p. 33-38, 2002.

2 BALMBRA, R.R.; CLUNIE, J.S.; GOODMAN, J.F.; CORKILL, J.M. EFFECT OF TEMPERATURE ON MICELLE SIZE OF A HOMOGENEOUS NON-IONIC DETERGENT. Transactions of the Faraday Society, v. 58, n. 476, p. 1661-1667, 1962.

3 BARNES, H.A. Thixotropy - A review. Journal of Non-Newtonian Fluid Mechanics, v. 70, n. 1-2, p. 1-33, 1997

4 BARNES, H.A.; HUTTON, J.F.; WALTERS, K. An Introduction to Rheology, Amsterdam: ELSEVIER Science Publishers B.V., 1998, p. 1-35.

5 BERTELLI, D.; PLESSI, M.; SABATINI, A.G.; LOLLI, M.; GRILLENZONI, F. Classification of Italian honeys by midinfrared diffuse reflectance spectroscopy (DRIFTS). Food Chemistry, v. 101, n. 4, p. 1565-1570, 2007.

6 BEZZOBOTNOY, V.Y.; BORBELY, S.; CSER, L.; FARAGO, B.; GLADKIH, I.A.; OSTANEVICH, Y.M.; VASS, S. TEMPERATURE AND CONCENTRATION-DEPENDENCE OF PROPERTIES OF SODIUM DODECYL-SULFATE MICELLES DETERMINED FROM SMALL-ANGLE NEUTRON-SCATTERING EXPERIMENTS. Journal of Physical Chemistry, v. 92, n. 20, p. 5738-5743, 1988.

7 BHANDARI, B.; D'ARCY, B.; CHOW, S. Rheology of selected Australian honeys. Journal of Food Engineering, v. 41, n. 1, p. 65-68, 1999.

8 BOGDANOV, S.; JURENDIC, T.; SIEBER, R.; GALLMANN, P. Honey for Nutrition and Health: A Review. Journal of the American College of Nutrition, v. 27, n. 6, p. 677-689, 2008.

9 DE ALMEIDA-MURADIAN, L.B.; STRAMM, K.M.; ESTEVINHO, L.M. Efficiency of the FT-IR ATR spectrometry for the prediction of the physicochemical characteristics of Melipona subnitida honey and study of the temperature's effect on those properties. International Journal of Food Science and Technology, v. 49, n. 1, p. 188-195, 2014.

10 HAMMOUDA, B. Clustering in polar media. Journal of Chemical Physics, v. 133, n. 8, p., 2010.

11 HAMMOUDA, B. Temperature Effect on the Nanostructure of SDS Micelles in Water. Journal of Research of the National Institute of Standards and Technology, v. 118, n., p. 151-167, 2013.

12 NAGAI, T.; INOUE, R.; KANAMORI, N.; SUZUKI, N.; NAGASHIMA, T. Characterization of honey from different floral sources. Its functional properties and effects of honey species on storage of meat. Food Chemistry, v. 97, n. 2, p. 256262, 2006.

13 PEREIRA, E.A.; JOS’EDEMELOQUEIROZ, A.; FIGUEIR`EDO, R.M.F.D. Comportamento reológico de mel da abelha uruçu (Melipona scutellaris, L.). Revista Ciencias Exatas e Naturais, v. 5, n. 2, p. 179-186, 2003.

14 SILVA, L.R.; VIDEIRA, R.; MONTEIRO, A.P.; VALENTAO, P.; ANDRADE, P.B. Honey from Luso region (Portugal): Physicochemical characteristics and mineral contents. Microchemical Journal, v. 93, n. 1, p. 73-77, 2009.

15 SILVA, R.C.D.; LOH, W. Effect of additives on the cloud points of aqueous solutions of ethylene oxide-propylene oxideethylene oxide block copolymers. Journal of Colloid and Interface Science, v. 202, n. 2, p. 385-390, 1998.

16 SVEČNJAK, L.; BILIŠKOV, N.; BUBALO, D.; BARIŠIĆ, D. Application of Infrared Spectroscopy in Honey Analysis Agriculturae Conspectus Scientificus, v. 76, n. 3, p. 191-195, 2011.

17 TSENG, I.H.; WATSON, S.S.; SUNG, L.P. Characterizing the dynamic behavior of nano-TiO2 agglomerates in suspensions by photocorrelation spectroscopy. Journal of Nanoparticle Research, v. 13, n. 5, p. 2195-2204, 2011. 
18 VIUDA-MARTOS, M.; RUIZ-NAVAJAS, Y.; FERNANDEZ-LOPEZ, J.; PEREZ-ALVAREZ, J.A. Functional Properties of Honey, Propolis, and Royal Jelly. Journal of Food Science, v. 73, n. 9, p. R117-R124, 2008.

19 WHITE, J.W., JR. Honey. Adv Food Res, v. 24, n., p. 287-374, 1978.

\section{AGRADECIMENTOS}

Os autores agradecem à FAPEMIG (APQ-4432-5.02/07, APQ-01243-10, APQ-0064512), ao CEFET-MG, ao CNPq (DT-310145/2011-2, MCT/CNPq-NANO: 550321/2012-8) e à UFJF (PROPESQ/PROPG) pelo suporte financeiro. 